\title{
OPERADORES EN LAS EXGLAMATIVAS CON ARTÍCULO DETERMINADO DE VALOR CUANTITATIVO
}

\section{INTRODUCCIÓN}

Hay en la gramática de las lenguas naturales oraciones cuyas unidades léxicas manifiestan, de alguna manera, la formación sintáctica e interpretación semántica de las mismas. Junto a éstas, se dan sin embargo otras que carecen de elemento léxico alguno que sugiera cómo están sintáctica y semánticamente formadas. Por ejemplo, en español, como es sabido, coexisten estructuras exclamativas con un elemento cuantificador ' $\mathrm{Qu}$-', como en el ejemplo (1), con estructuras en las que no hay un cuantificador explícito, como en $(2)^{1}$ :

(1) a. ¡Cuántas cervezas consume la gente en este país!

b. ¡Cuánta cerveza consume la gente en este país!

(2) a. ¡Las cervezas que consume la gente en este país! b. ¡La cerveza que consume la gente en este país!

Las oraciones del ejemplo (2) admiten una interpretación cuantitativa análoga a la de las oraciones del ejemplo (1). Concretamente, en (2), cabe interpretar los sintagmas nominales las cervezas y la cerveza como "las muchas cervezas" y "la mucha cerveza", respectivamente.

Las oraciones de (2) plantean la cuestión de cómo llega el hablante a adscribir a tales secuencias de sonidos un valor cuantitativo. Dado que las exclamativas cuantitativas de artículo determinado del español no tienen ningún elemento fonético que

1 Véase, por ejemplo, la mención que hace de las mismas Salvador FERNÁNDEZ Ramírez en su Gramática española, Revista de Occidente, Madrid, 1950, p. 319. 
indique cantidad, hay que suponer que el hablante se vale de principios generales que son parte de la gramática que ha internalizado para formarlas. Como los principios que entran en la formación sintáctica y semántica de estas oraciones son principios de las gramáticas de las lenguas naturales en general, el estudio de las construcciones de una lengua particular queda siempre enmarcado dentro de la gramática comparada.

Adviértase que, a pesar de que las oraciones (2) no llevan un operador ' $\mathrm{Qu}$-' representado fonéticamente, tanto los contextos sintácticos en donde aparecen como la intepretación semántica de estas exclamativas hace suponer que, a un nivel abstracto de representación, las estructuras de (2) sí tienen un operador ' $Q u^{-}$'. Por ejemplo, las oraciones exclamativas introducidas por el artículo determinado del español pueden aparecer como oraciones subordinadas con la misma clase de verbos que permiten las exclamativas con un elemento ' $Q u^{-}$' fonéticamente representado: 'imaginar', 'darse cuenta', 'saber', 'ver', etcétera.

(3) a. ¡No sabes/no te imaginas cuántos pares de zapatos encontraron en la casa!

b. ¡No sabes/ no te imaginas los pares de zapatos que encontraron en la casa!

Y en ambos casos, la interpretación exclamativa de estas oraciones sólo es posible en el contexto de la negación, el de la pregunta o algún otro operador:

(4) a. Sé cuántos pares de zapatos encontraron en la casa.

b. Sé los pares de zapatos que encontraron en la casa.

Con entonación neutral, ni (4a) ni (4b) es oración exclamativa.

La formación sintáctica de las oraciones de (2) no deja de ser más intrincada, hasta cierto punto, que la de las oraciones de (1). En este trabajo presentaré argumentos en favor de la presencia de dos operadores nulos en las exclamativas de tipo (2): un operador 'Qu-' en la oración introducida por que, y un cuantificador en el sintagma nominal que la precede.

Desde el punto de vista sintáctico, el comportamiento de la oración introducida por que es, como veremos, el propio de las estructuras dotadas de un operador ' $Q \mathrm{u}^{-}$' con contenido fonético. En este sentido, analizar las exclamativas de (2) como estructuras dotadas de un operador ' $Q \mathbf{u}^{-}$' $\sin$ representación fonética 
es la hipótesis más simple dentro del marco teórico de la gramática generativa en el que centro mi propuesta. Tal como se analizan las oraciones dotadas de un operador léxico, argüiremos que el operador nulo 'Qu-' de las exclamativas de (2) se desplaza a la posición del especificador de O' donde aparece el correspondiente operador léxico ${ }^{2}$. Con todo, la distribución del operador nulo de estas oraciones es, tal como demostraremos, más restringida que la del operador 'Qu-' de las oraciones de relativo no cuantificadas.

El segundo operador nulo que proponemos atañe al sintagma nominal de más a la izquierda de la oración, específicamente; así, en (4b), al sintagma los pares de zapatos. Nuestro análisis postulará la existencia de un cuantificador sin representación fonética (muchos) ( = 'los [muchos] pares de zapatos'), el cual, en el componente de la estructura lógica de la gramática, sube a una posición interna del sintagma nominal por cuestiones de alcance, tal como se asume en estructuras con cuantificadores léxicos. Las pruebas empíricas que daremos justificando esta operación de movimiento "invisible"' son un calco de las restricciones a las que está sujeta en español la extracción de elementos con representación fonética fuera de los sintagmas nominales.

Movimiento DE UN OPERADOR NULO EN EL COMPONENTE SINTÁCTICO

Los principios que rigen el movimiento en el componente sintáctico de la gramática afectan tanto a elementos con representación fonética como a los elementos que no se pronuncian. Los hechos que presentamos a continuación sirven como indicio de que hay un elemento 'Qu-' que se desplaza en las exclamativas de artículo determinado cuantificadas del español ${ }^{3}$. Considérense los siguientes ejemplos:

2 Se asume que el especificador de O" es la posición más externa de $O$ ".

${ }^{3}$ Adviértase que mi análisis sólo incluye a las exclamativas de artículo determinado con valor de cantidad que tienen una frase nominal como antecedente. No estudio aquí ni el comportamiento sintáctico de las exclamativas de tipo (i) y (ii), ni tampoco el de (iii):

(i) ¡Lo alta que está esta niña!

(ii) ¡Lo que ha llorado este chico!

(iii) ¡La de gente que ha llamado hoy por teléfono! 
(5) a. *[Cuántos libros [ te han hecho [la propuesta de O' O NP [publicar $\left.\left.\left.\left.\mathrm{h}_{\mathrm{i}}\right]\right]\right]\right]$ $\mathrm{O}$

b. *[Los libros ${ }_{\mathrm{i}}\left[\mathrm{Op}_{\mathrm{i}}\right.$ que [te han hecho [la propuesta de

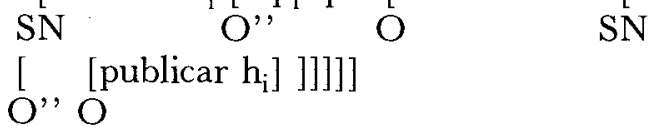

Las oraciones de (5) no son secuencias gramaticales. En (5a), el sintagma cuántos libros se ha movido desde la posición marcada por ' $h$ ', su huella, a través de un sintagma nominal 'la propuesta de...' En (5b), no hay ningún sintagma fonéticamente representado que se desplace. Con todo, la estructura sintáctica de (5b) es idéntica a la de (5a). Es pues razonable suponer que cualquiera que sea el principio que excluye a (5a) en español, excluye también a (5b). De modo que el paralelismo entre (5a) y (5b) proporciona un argumento a favor del supuesto de que hay un elemento que se desplaza en la oración introducida por que $e^{4}$.

Cabe hacer el mismo tipo de razonamiento a propósito de los ejemplos de (6):

(6) a. ¡[Cuántos libros [no se explicaban [a quién no sabían [por qué [regalar $\left.\left.\left.\left.\left.\mathrm{h}_{\mathrm{i}}\right]\right]\right]\right]\right]$ !

b. i[Los libros $\left[\mathrm{O}_{\mathrm{i}}\right.$ que [no se explicaban [a quién no sabían [por qué [regalar $\left.\left.\left.\left.\left.\left.\mathrm{h}_{\mathrm{i}}\right]\right]\right]\right]\right]\right]$ !

Una vez más, el movimiento de la posición marcada por la huella $h_{i}$ produce agramaticalidad tanto en (6a) como en (6b). Si no existiera en la estructura sintáctica de (6b) un elemento nulo que se desplaza a la oración de O' introducida por que, la imposibilidad de (5b) y (6b) quedaría sin explicar.

Ahora bien, la distribución del operador nulo ' $\mathrm{Qu}$-' que postulamos en las exclamativas de artículo determinado del español es mucho más restringida que la del operador fonéticamente representado cuántos. Tal contraste no es sorprendente. Es de espe-

${ }^{4}$ Las posibilidades de traslado de una categoría, desde su posición subyacente hasta la que ocupa en la estructura que se obtiene, están regidas por el principio de la subyacencia. Dado que nuestra intención es sólo la de mostrar el paralelismo de las posibilidades de traslado de un elemento ' $Q \mathbf{u}$-' léxico y de otro no léxico, no consideramos relevante reproducir aquí la formalización de este principio. 
rar que una teoría gramatical que hace uso de categorías fonéticamente nulas tenga principios generales que restrinjan la aparición de las mismas. Naturalmente, en el aprendizaje de las lenguas, el niño no cuenta con piezas léxicas que lo induzcan a detectar la presencia de categorías vacías en las oraciones. Por tanto, no podrían ser las categorías nulas parte de las lenguas naturales si no existieran principios de carácter universal que determinaran las condiciones bajo las cuales pueden aparecer. En el ámbito de las categorías sin representación fonética, la tarea del gramático es la de determinar cuáles son éstas y qué principios las rigen. En el caso concreto que nos ocupa, si hay contextos sintácticos que separan el comportamiento de las oraciones de (2) del de las de (1), tal cosa vendría a apoyar la hipótesis del movimiento de una categoría nula en las oraciones de (2).

Comparemos la distribución de (1) y (2) en los siguientes contextos sintácticos:

(7) a. ¡No sabes en cuántos armarios han encontrado pares de zapatos!

b. ¡No sabes en los armarios que han encontrado pares de zapatos!

La oración (7b) no admite una interpretación cuantificada. Concretamente, el sintagma preposicional en los armarios no equivale aquí a 'los muchos armarios'; (7b) sólo puede interpretarse aludiendo a la cualidad de los armarios: 'en la clase de armarios...' Obsérvese que, contrariamente a los ejemplos de (2), la posición vacía de la oración de relativo que se computa mentalmente en (7b) es la de un sintagma preposicional; es decir, la representación sintáctica que le correspondería a (7b) es la esquematizada en $(8)^{5}$ :

(8) ... [ que $\left[\right.$ [pro] han encontrado pares de zapatos $\left.\left.\left[\mathrm{h}_{\mathrm{i}}\right]\right]\right]$ $\mathrm{O}^{\prime \prime} \mathrm{O}^{\prime}$ SP

En otras palabras, parece que el operador nulo de las exclamativas cuantificadas de artículo determinado no puede ser parte de un sintagma preposicional.

Considérense ahora las oraciones de (9):

${ }^{5}$ En la esquematización de (6), '[pro]' representa al pronombre sujeto que no tiene, en este caso, representación fonética. 
(9) a. ¡No te imaginas cuántas cosas le preocupan!

b. ¡No te imaginas las cosas que le preocupan!

De nuevo, la interpretación de (9a) y (9b) no es la misma. Contrariamente al caso de la exclamativa (9a), con un 'Qu-' explícito (9b) sólo admite una interpretación de cualidad: 'el tipo de cosas...' En contextos como (9b), la cantidad en español va expresada por medio del sintagma nominal: la de cosas, o de la [cantidad] de cosas. . . ; es de suponer que la expresión la de cosas es una variante elíptica de la de la cantidad de cosas.

Adviértase que no existe semejante restricción en las oraciones de relativo regulares en las que, supuestamente, hay también un operador ' $Q \mathrm{u}^{-}$' nulo:

(10) Nos contó las cosas que le preocupaban.

Evidentemente (10) es una oración bien formada del español.

Un contraste parecido puede verse en ejemplos del siguiente tipo:

(11) a. !Qué barbaridad! ¡Cuántos actores hacen el ridículo!

b. !Qué babaridad! ¡Los actores que hacen el ridículo!

La interpretación cuantitativa de (11b) resulta muy difícil o imposible.

Nótese que el lugar "vacío" de la oración de relativo que se computa tanto en (9b) como en (11b) es el del sujeto. Por alguna razón, el operador nulo de las oraciones exclamativas de tipo (2) produce oraciones mal formadas cuando se origina en la posición de sujeto. Una vez más, las oraciones de relativo regulares no están sujetas a esta restricción:

(12) Los actores que hacen el ridículo no suelen ser buenos.

Conviene señalar que cuando los sujetos son semánticamente objetos, en otras palabras, cuando se trata de sujetos "derivados", la restricción que acabamos de señalar a propósito de (9) y (11) no está vigente:

(13) a. ¡Hay que ver cuánta gente cabe en esta sala! b. ¡Hay que ver la gente que cabe en esta sala! 
Lo mismo ocurre, naturalmente, en las pasivas:

(14) a, ¿Has visto cuánta gente ha sido detenida?

b. ¿Has visto la gente que ha sido detenida?

Tanto en (13b) como en (14b), el operador nulo se originaría en la posición posverbal de objeto, no en la posición preverbal de sujeto, tal como queda sugerido en el esquema de (15):

$$
\ldots \mathrm{O}^{,}, \mathrm{O}_{\mathrm{i}} \text { que }\left[\ldots\left[\mathrm{O}\left[\mathrm{VV}\left[\mathrm{t}_{\mathrm{i}}\right] \ldots\right]\right]\right]
$$

No cabe duda de que cualquiera que sea la restricción que excluya el movimiento del operador nulo 'Qu-' desde la posición sujeto, no ha de afectar a los objetos; los ejemplos (13b) y (14b) son, en efecto, casos de movimiento a partir de la posición de objeto.

Un último argumento a favor de la hipótesis de que las exclamativas de tipo (2) tienen un operador nulo 'Qu-' que se desplaza a O' en el componente sintáctico es la posibilidad de construir oraciones que son "parasitarias" de un elemento 'Qu-'.

(16) a. ¡Vaya! ¡Has devuelto los libros sin mirarlos!

b. Vaya! ¡Has devuelto los libros sin mirar!

c. ¿Qué barbaridad! ¡Cuántos libros has devuelto sin mirar!

d. ¡Qué barbaridad! ¡Los libros que has devuelto sin mirar!

Adviértase que el ejemplo (16b) no admite la interpretación de (16a); es decir, no cabe interpretar la expresión adverbial "sin mirar" sobreentendiendo el objeto pronominal 'los'. En cambio, tanto (16c) como (16d) permiten, marginalmente, una interpretación en la que se incluye la información del objeto del verbo: 'sin mirar los libros'. Casos como (16c) y (16d) se utilizan en la gramática generativa como prueba sintáctica de la presencia de un operador 'Qu-'. Si bien (16c) y (16d) son, ambas, oraciones poco naturales, también es verdad que son mucho mejores que (16b), en el sentido apuntado antes. Por lo tanto, es razonable suponer la presencia de un operador 'Qu-' tanto en una como en otra.

No nos ocuparemos aquí de los principios que pudieran dar razón de la distribución limitada del operador nulo de las exclamativas con valor cuantitativo del español. Hasta el momento, las limitaciones de aparición de los operadores nulos 'Qu-' han 
sido estudiadas en detalle sólo para el inglés. Con excepción de las oraciones de relativo, las construcciones para las que se ha postulado en inglés un operador nulo son todas oraciones de infinitivo ${ }^{6}$. Sin embargo, las gramáticas de las lenguas románicas como el español difieren considerablemente de las de lenguas como el inglés en este ámbito de la gramática. Nos limitaremos a señalar que, aun así, algunas de las restricciones de aparición del operador nulo de las oraciones exclamativas del español se dan también en las construcciones de infinitivo del inglés en las que se ha postulado un operador sin manifestación fonética; en particular, la de que el operador nulo no puede ligar una variable en posición de sujeto?

\section{El ANTECEDENTE DEL OPERADOR}

El operador de la oración introducida por que en las exclamativas de tipo (2), por ser nulo, no tiene de por sí contenido semántico. Los operadores nulos, en general, requieren un antecedente para ser interpretados semánticamente. El antecedente del operador de las oraciones de (2) es el sintagma nominal que le precede: las cervezas. Adviértase que en la interpretación de (2) hay que incluir un argumento que actúe de complemento del verbo; por ejemplo, en (2a), el sintagma nominal las cervezas. La presencia de un operador nulo justifica precisamente el que exista esta información.

Ahora bien, los elementos que están léxicamente presentes en estos sintagmas nominales, por sí solos, harían, de los mismos,

${ }^{6}$ Las construcciones de infinitivo que se analizan como estructuras con operador nulo para el inglés incluyen: (a) oraciones adjetivas del tipo: María es demasiado inteligente como para. . . ; (b) complementos de adjetivos: Juan es difícil de complacer; oraciones como: Compraron el libro para. . . ; y, por supuesto, las oraciones adverbiales que mencionamos a propósito de (16c).

${ }^{7}$ Hay una propiedad más que debemos incluir aquí. Parece que el operador de las exclamativas del español, como los operadores de cierta clase de oraciones de infinitivo del inglés, resiste el desplazamiento a partir de oraciones subordinadas. Obsérvese, por ejemplo, que es más fácil sacar la interpretación cuantitativa en el ejemplo (i) que en el (ii); y, a su vez, esta interpretación es más accesible en (ii) que (iii):

(i) ¡Los factores que hay que tener en cuenta!

(ii) ¡Los factores que nos aconsejaron que tuviéramos en cuenta!

(iii) ¡Los factores que nos aconsejaron advertir a la gente que tuviera en cuenta!

Esto, sin duda, añade anormalidad a los ejemplos (5b) y (6b), ya de por sí, excluidos por el principio de subyacencia. 
sintagmas nominales definidos no cuantificados. Sin embargo, la interpretación semántica de un sintagma nominal como el de (2a) no es la de un sintagma definido no cuantificado, sino la de un sintagma cuantificado, algo así como: 'la cantidad x de cervezas', donde ' $x$ ' se interpreta como una cantidad grande. Es más, el sintagma nominal introducido por el artículo definido en estas exclamativas no se comporta como un sintagma definido. Sintácticamente, las oraciones exclamativas de artículo determinado se comportan como los sintagmas indefinidos. Así, con el verbo haber, un sintagma definido como el de (17a) produce una oración anormal; en cambio (17b), (17c) y (17d) son oraciones del español bien formadas:

(17) a. *Hay la gente aquí

b. Hay mucha gente aquí

c. ¡La gente que hay aquí!

d. ¡Cuánta gente hay aquí!

Podemos suponer que la restricción que hace que 'haber' tenga que tomar complementos indefinidos en casos como (17a) opera en el componente de la forma lógica de la gramática. Si esto es así, el contraste entre (17a) y (17c) sugiere que el complemento de 'haber' de (17c), en la forma lógica, es indefinido. Nótese que 'haber', en (17c), tiene por complemento la huella del operador 'Qu-' que se desplaza en la sintaxis: '[ ... [ Op $p_{i}$ que [hay $h_{i}$ aquí]]]'. Ahora bien, el operador nulo 'Qu-', que liga la variable ' $h$ ' en esta estructura, adquiere interpretación a partir de su antecedente: el sintagma nominal cuantificado mucha gente; semánticamente, el operador es, por tanto, indefinido. Dado que el operador es de carácter indefinido, la variable que liga también habrá de serlo. De manera que la gramaticalidad de (17c) no es más que un reflejo de la gramaticalidad de (17d); en ambos casos, 'haber' tiene complementos indefinidos al nivel de la forma lógica.

De hecho, el sintagma nominal que sirve de antecedente al operador nulo de las exclamativas cuantificadas sin operador léxico del español no puede ir introducido por cualquier especificador definido. Sólo el artículo definido permite la cuantificación, tal como se ve en el siguiente contraste:

(18) a. ¡Los libros que lees!

b. ¡Estos libros que lees! 
Está claro que (18a) puede interpretarse cuantitativamente, pero no $(18 b)$.

Es más, hay determinados contextos sintácticos en los que el artículo determinado no se presta, en español, a la interpretación cuantificada de estas oraciones; compárense los siguientes ejemplos:

(19) a. ¡No te imaginas los talleres de automóvil que han cerrado últimamente!

b. ¡Pues tú no te imaginas los $[\mathrm{N}]$ de productos químicos que han abierto!

Es mucho más difícil, si no imposible, interpretar cuantitativamente (19b) que (19a) ${ }^{8}$. El contraste entre (19a) y (19b) es, en todo, paralelo al de (18a) y (18b); a saber, los demostrativos impiden la interpretación cuantitativa de estas oraciones.

Es importante señalar que el nombre que sirve de núcleo al sintagma nominal que precede a la oración de que en (19d) es elíptico: 'los [N] de productos químicos'; la interpretación semántica de este nombre nulo viene dada por el nombre talleres del sintagma nominal de la oración (18a): los talleres de automóvil. La presencia de un nombre nulo en este tipo de sintagmas nominales del español tiene su costo. En particular, el artículo determinado, en este caso, adquiere la fuerza de un demostrativo, tal como han intuido, de una u otra forma, casi todos los gramáticos del español $^{9}$. Volveremos más adelante a la cuestión del artículọ determinado en estas construcciones.

Con todo, los argumentos más fuertes para postular un cuantificador nulo en los sintagmas nominales de las exclamativas de (2) son de carácter sintáctico. A diferencia de lo que ocurre en las exclamativas de cuantificador léxico (cuántos/cuánto), la presencia de cierta clase de argumentos dentro del sintagma nominal de las exclamativas del tipo (2) impide la interpretación cuantificada de estas oraciones. Obsérvense los siguientes contrastes:

(20) a. ¡Qué barbaridad! ¡El dinero que se ha gastado!

b. ¿Qué barbaridad! ¡El dinero de su familia que se ha gastado!

${ }^{8}$ Adviértase que la interpretación cuantitativa no plantea problemas en oraciones como:

(i) ¡La de industrias químicas que han abierto!

${ }^{9}$ Para un resumen crítico del tratamiento que hacen los gramáticos del español del artículo con valores anafóricos, véase JosÉ M. BRUCART, La elisión sintáctica en español, Universitat Autonoma de Barcelona, Bellaterra, 1987, pp. 221-228. 
La presencia del genitivo posesivo de su familia impide la interpretación cuantificada de (20b). Sin embargo, los genitivos posesivos no afectan a la formación de las exclamativas de cantidad que llevan un operador léxico cuantitativo 'Qu-':

(21) ¿Qué barbaridad! ¡Cuánto dinero de su familia se ha gastado!

También los genitivos agentes, en determinadas condiciones, impiden la interpretación cuantificada de las exclamativas con operador nulo:

(22) a. ¡Qué barbaridad! ¡Los cuadros de tema religioso que ha acumulado este museo!

b. ¡Qué barbaridad! ¡Los cuadros de tema religioso de El Greco que ha acumulado este museo!

Una vez más, la interpretación cuantitativa de (22b) es muy difícil o imposible de sacar. Adviértase que las exclamativas cuantificadas que llevan un elemento léxico 'Qu-' no están sujetas a semejante restricción:

(23) ¡Qué barbaridad! ¡Cuántos cuadros de tema religioso de El Greco ha acumulado este museo!

En cambio, otros argumentos del nombre no afectan a la interpretación cuantitativa de estas oraciones:

(24) a. ¡La gente de El Salvador que ha muerto en esta guerra!

Dado que tanto los genitivos posesivos como los genitivos agentes no afectan a las exclamativas que llevan cuántos y cuánto, la restricción que estos genitivos imponen a las exclamativas de operador nulo no puede ser de carácter semántico. Si lo fuera, estos genitivos habrían de tener los mismos efectos en los dos tipos de exclamativas, no únicamente en las de operador nulo.

Debe mencionarse también que estas dos clases de genitivos impiden el desplazamiento de los complementos del nombre. Por ejemplo, las preguntas (25b) y (25c), contrariamente a la (25a), no están bien formadas:

(25) a. ¿De qué temas han expuesto varios cuadros? b. ¿ंDe qué temas han expuesto varios cuadros de Goya? 
c. ${ }^{*}$ ¿De qué temas han expuesto varios cuadros de este coleccionista?

Es un hecho crucial que los mismos genitivos que impiden que se desplace un complemento ' $Q \mathrm{u}^{-}$' del nombre en las preguntas, interfieran también con la interpretación cuantificada de las oraciones exclamativas (22) y (23). Obsérvese que el tipo de genitivo que lleva la exclamativa (24a) no interfiere con la extracción de un complemento del nombre:

(26) ¿De qué obra $\mathrm{i}_{\mathrm{i}}$ han reseñado [una traducción $\mathrm{h}_{\mathrm{i}}$ de El Salvador]?

Las restricciones que acabamos de señalar indican que existe movimiento en el interior del sintagma nominal de las exclamativas que estamos analizando. Puesto que se trata de expresiones cuantitativas, lo más razonable es suponer que el sintagma nominal introducido por el artículo determinado tiene un cuantificador fonéticamente nulo, y que éste sube a una posición desde la que puede abarcar el sintagma nominal entero ${ }^{10}$.

La operación de desplazamiento de cuantificadores tiene lugar en el componente de la forma lógica de la gramática, y da lugar a representaciones en las que el cuantificador liga una variable, su huella. Ahora bien, los principios de rección que operan sobre las huellas creadas a partir de desplazamientos en el componente de la forma lógica son los mismos que operan sobre las huellas originadas en la estructura sintáctica. De ello, cabe concluir que la razón por la cual ciertos argumentos del nombre impiden la interpretación cuantitativa de los sintagmas nominales que aquí estudiamos es la misma que la que impide la extracción de argumentos fuera del sintagma nominal. En ambos casos, la relación estructural que media entre el operador desplazado y su variable tiene demasiada estructura intermedia como para estar bien formada. Hay que señalar que la relación formal que ha de mediar entre las huellas y sus antecedentes es estrictamente local. En los sintagmas nominales del español, los genitivos posesivos y ciertos agentes interrumpen la relación de localidad a la que están sujetas las huellas en general.

${ }^{10}$ Esta operación vendría a ser una aplicación más de la regla de subida de cuantificadores propuesta por ROBERT MAY, Logical form. Its structure and derivation, MIT Press, Cambridge, 1985. 


\section{ESTRUCTURA}

Nos corresponde ahora reunir los criterios a partir de los cuales se forman las exclamaciones con artículo determinado de valor cuantitativo en español.

La estructura sintáctica de las oraciones exclamativas que hemos examinado es, esencialmente, la de las oraciones de relativo. La oración introducida por que tiene un operador nulo ' $\mathrm{Qu}$-' que facilita la relación de esta oración con el sintagma nominal que le precede. La relación que media entre ambos es la de una predicación. Semánticamente, el operador nulo 'Qu-' de la oración con que adquiere un valor cuantitativo a partir de su antecedente; dado que éste, el sintagma nominal que le precede, es una estructura de cuantififación, la interpretación del operador ' $Q \mathrm{u}^{-}$' es también la de un sintagma cuantificado. Esquemáticamente, estas relaciones pueden expresarse así:

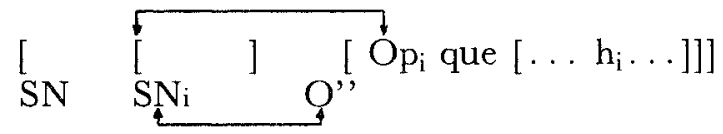

Una diferencia importante entre las oraciones de relativo y las exclamativas cuantitativas de artículo determinado es que sólo las segundas aparecen como oraciones independientes. Es decir, tal como se asume generalmente, las oraciones de relativo son sintagmas nominales que hacen las funciones de sujeto o complemento. En cambio, las oraciones exclamativas de artículo determinado, a pesar de tener la estructura de los sintagmas nominales, se presentan como oraciones autónomas ${ }^{11}$. Este hecho podría estar relacionado con el carácter proposicional de esta clase de exclamativa. Como vimos anteriormente, las exclamativas cuantitativas de artículo determinado pueden aparecer como oraciones subordinadas con ciertos verbos que seleccionan preguntas: imaginar, darse cuenta, no saber, etc. Es verdad que ni preguntar ni preguntarse permiten exclamativas; las oraciones me pregunto cuánta gente cabe aqui, y pregunté cuánta gente cabía aquí son preguntas, no exclamaciones. Esta restricción, sin embargo, parece deberse a la semántica de los verbos de pregunta, específicamente. Evidentemente, la se-

11 Nótese que esta clase de exclamativas no pueden ir en infinitivo:

(i) No sé cuántos libros leer.

(ii) No sé los libros que leer.

En (i), la oración subordinada no puede ser exclamativa, y en (ii) es una secuencia agramatical. 
mántica de los verbos de pregunta que toman exclamativas ha de ser compatible con la semántica de las propias exclamativas.

Una característica esencial de la clase semántica de los verbos de pregunta es que seleccionan complementos proposicionales encabezados por un operador $\left[+Q \mathbf{u}^{-}\right]$; esto implica que tanto en: me preguntó qué hora era como en me preguntó la hora, el complemento de preguntar es de carácter proposicional. En el nivel de la forma lógica, los complementos de verbos de pregunta han de interpretarse como complementos [ + Qu-]; la clase de complementos [ + Qu- incluye, no sólo las preguntas, sino las exclamaciones. Ahora bien, las exclamativas cuantitativas de artículo determinado carecen, de nuevo, de un elemento léxico '[ +Qu- $]$ ' que sirva a las necesidades de complementación de esta clase de verbos. Una vez más, nos enfrentamos al problema de detectar cuál será el elemento [ + Qu-] que encabece la estructura de las exclamativas de artículo determinado. Existe, no obstante, en las exclamativas de tipo (2) un elemento léxico cuyas características sintácticosemánticas son particularmente idóneas para que asuma el rasgo [+Qu-] que se precisa: el artículo determinado.

Tal como establecimos anteriormente, ni los demostrativos, ni el artículo determinado con valor anafórico, sirven para introducir exclamativas cuantificadas sin elemento léxico ' $Q u^{-}$'. Lo natural es suponer que es precisamente el artículo determinado el elemento que absorbe el rasgo [ + Qu-] que seleccionan los verbos de pregunta. Semánticamente, esta hipótesis es muy plausible. A diferencia de los demostrativos, el artículo determinado sin valor demostrativo carece de semántica propia. Sintácticamente, nuestra hipótesis implicaría que el artículo determinado encabeza la construcción. En resumen, las propiedades sintácticas y semánticas de las exclamativas cuantitativas de artículo determinado son idénticas a las de las exclamativas de cuantificador [ $\left.+\mathrm{Qu}^{-}\right]$.

El que las oraciones exclamativas cuantificadas de artículo determinado sean un calco de las exclamativas léxicamente cuantificadas por cuántos y cuánto es bastante natural. Es un fenómeno común de las lenguas naturales el que exista la contrapartida no léxica de una estructura sintática léxica.

Esther TORREGo 